\title{
Gene expression in nontumoral liver tissue and recurrence-free survival in hepatitis $C$ virus- positive hepatocellular carcinoma
}

\author{
Masato Tsuchiya ${ }^{1,3}$, Joel S Parker ${ }^{2}$, Hiroshi Kono ${ }^{3}$, Masanori Matsuda ${ }^{3}$, Hideki Fujii ${ }^{3}$, Ivan Rusyn ${ }^{*}$
}

\begin{abstract}
Background: The goal of this study was to understand gene expression signatures of hepatocellular carcinoma (HCC) recurrence in subjects with hepatitis C virus (HCV) infection. Recurrence-free survival (RFS) following curative resection of HCC in subjects with HCV is highly variable. Traditional clinico-pathological endpoints are recognized as weak predictors of RFS. It has been suggested that gene expression profiling of HCC and nontumoral liver tissue may improve prediction of RFS, aid in understanding of the underlying liver disease, and guide individualized patient management. Frozen samples of the tumors and nontumoral liver were obtained from 47 subjects with HCV-associated HCC. Additional nontumoral liver samples were obtained from HCV-free subjects with metastatic liver tumors. Gene expression profiling data was used to determine the molecular signature of HCV-associated HCC and to develop a predictor of RFS.

Results: The molecular profile of the HCV-associated HCC confirmed central roles for MYC and TGF $\beta 1$ in liver tumor development. Gene expression in tumors was found to have poor predictive power with regards to RFS, but analysis of nontumoral tissues yielded a strong predictor for RFS in late-recurring ( $>1$ year) subjects. Importantly, nontumoral tissue-derived gene expression predictor of RFS was highly significant in both univariable and multivariable Cox proportional hazard model analyses.

Conclusions: Microarray analysis of the nontumoral tissues from subjects with HCV-associated HCC delivers novel molecular signatures of RFS, especially among the late-recurrence subjects. The gene expression predictor may hold important insights into the pathobiology of HCC recurrence and de novo tumor formation in cirrhotic patients.
\end{abstract}

\section{Background}

Hepatocellular carcinoma (HCC) is the fifth most common cancer worldwide by annual incidence and the third leading cause of cancer death [1]. Wide geographic variation in age-adjusted incidence and death rates is well recognized [2]. Most alarming is the fact that ageadjusted incidence and death rates for cancer of the liver and intrahepatic bile duct show a statistically significant increasing trend in the past three decades in the USA and many other countries, even though other major cancers are on a decline [3]. This rise is being attributed, at least in part, to an increase in incidence of hepatitis $\mathrm{C}$ virus $(\mathrm{HCV})$ infections and non-alcoholic

\footnotetext{
* Correspondence: iir@unc.edu

'Department of Environmental Sciences and Engineering, Gillings School of Global Public Health, University of North Carolina, Chapel Hill, NC, USA
}

steatohepatitis, pathological states that are also growing in the US population $[4,5]$. The resistance of HCC to existing treatments and the lack of biomarkers for early detection make it one of the deadliest cancers. Surgical resection, liver transplantation, and ablation by radiofrequency or ethanol injection are now conventional therapies at early disease stages. Even with these options, survival at 5 years is poor and ranges between $50 \%$ and $70 \%[6]$.

One of the key reasons for poor long-term survival in HCC is high incidence of recurrence, a complication that cannot be prevented effectively by new and existing therapies $[7,8]$. Many clinico-pathological features, such as tumor size, number of tumors in liver, capsule state, cell differentiation, venous invasion, and the extent of intrahepatic spreading are commonly used in clinical

\section{C) Biomed Central}


diagnosis as predictive risk factors for $\mathrm{HCC}$ recurrence and health prognosis in patients $[9,10]$. However, the prospective utility of these attributes for predicting recurrence-free survival (RFS) may be more limited as $\mathrm{HCC}$ is being diagnosed at earlier stages.

Several research groups have performed gene expression profiling of both tumor and nontumoral specimens and identified gene signatures of recurrence-free [11], or overall survival [12] in HCC patients who undergo tumor resection. These studies carry an exciting potential to open the field to more selective chemoprevention as a follow-up to surgical interventions since patients with greatest risk of death or recurrence can be potentially identified [12] and/or individualized therapies may be devised based on the molecular profiles of poor-prognosis markers of HCC $[13,14]$. At the same time, the issues of validation of the molecular signatures, the utility of target-gene list strategy for predicting early- versus late-recurrence, and the value of these studies for understanding pathobiology of the underlying liver disease in patients with HCC are still open for consideration [15].

Thus, our study focused on a well-defined cohort of HCC patients who were HCV positive (but free of hepatitis $B$ virus) and underwent tumor resection to conduct a comparative microarray-based gene expression profiling of tumor and nontumoral specimens. We report the molecular network signatures of HCV-associated HCC, as well as the outcome of the analysis of the predictive value of gene expression in tumor- and nontumoral tissue-derived samples. This study was not only successful in developing a highly predictive RFS signature for late recurrence from nontumoral samples, but also shows that this signature can be used for biological interpretation of the liver disease leading to recurrence.

\section{Results and Discussion}

\section{Clinico-pathological markers of RFS in HCV-associated HCC}

This study evaluated specimens (frozen tumor and/or nontumoral tissue) collected during liver tumor resection in (i) subjects with solitary tumors in liver that were a result of metastasis from other organs, and (ii) subjects manifesting HCC in combination with chronic $\mathrm{HCV}$ infection and absence of known tumors at other sites. Platelet count, alanine aminotransferase and fibrosis score were significantly different between the groups (Table 1). The HCC cohort was subdivided into nontumoral and tumor groups for the purposes of molecular analysis (see below). No differences between these two groups, or between each group and the entire cohort, exist based on the available clinical data. The univariable analysis of the prognostic power for various clinicopathological measurements available in this study showed that tumor stage, diameter and, to a lesser degree, multiplicity were statistically significant in predicting the length of RFS in subjects with HCV-associated HCC (Table 2). Other characteristics were not significant, including the type of the surgical procedure performed (e.g., lobular or segmental/partial hepatectomy). These results are consistent with the reports in the literature [16].

\section{Molecular profiling of HCV-associated HCC}

The importance of defining liver tumor biology that may aid in the development of new screening and treatment stratification programs to refine diagnosis and improve patient outcome is well recognized [15]. Thus, our first step was to evaluate gene expression data $(25,073$ transcripts) collected on individual tumor and nontumoral samples. Principal components analysis (Genomics Suite; Partek Inc., St. Louis, MO) was used to examine global variation in transcript abundance (Figure 1). The unsupervised analysis displayed separation of the samples between HCC tumor samples and nontumoral samples from subjects with HCC and metastatic liver tumors indicating that gene signatures indicative of HCC tumor biology may be explored.

To evaluate the biological features of $\mathrm{HCV}$-associated HCC we compared gene expression profiles of tumors and control (nontumoral tissue from metastatic liver tumor) samples. The analysis revealed 155 up- and 1,248 down-regulated genes whose expression levels were significantly different $(F D R=0)$. Next, functional pathway analysis was performed using significant genes (Additional file 1, Tables S2-3). Several pathways were identified as significantly up- or down-regulated in $\mathrm{HCV}$-associated HCC. Interestingly, the top-ranked network composed of up-regulated genes includes TGF $\beta 1$ and MYC as hubs (Additional file 2, Figure S1). Indeed, it has been suggested that chronic inflammation associated with HCV infection in liver facilitates TGF $\beta$ signaling cascade involving c-Jun $\mathrm{N}$-terminal kinase and plasminogen activator inhibitor 1 which facilitates fibrogenesis, promotes liver cirrhosis and increases the risk of development of HCC [17]. In addition, gene expression-based comparison between dysplastic nodules and early hepatocellular carcinomas identified the MYC oncogene as a plausible driver gene for malignant transformation of dysplastic nodules [18]. Thus, our data provides independent confirmation of and strengthens the evidence [19] for the key roles of TGF $\beta 1$ and MYC in the progression of $\mathrm{HCC}$ in patients with $\mathrm{HCV}$.

\section{Tumor-derived gene expression profiles and recurrence- free survival in HCV-associated HCC}

Recurrence of HCC is thought to arise due to intrahepatic metastasis, or the development of a second 
Table 1 Clinical characteristics of patients.

\begin{tabular}{|c|c|c|c|c|}
\hline Variables & $\begin{array}{l}\text { HCC Cohort } \\
(\mathrm{N}=47)\end{array}$ & $\begin{array}{l}\text { Nontumoral HCC cohort } \\
(\mathrm{N}=44)\end{array}$ & $\begin{array}{l}\text { Tumor HCC cohort } \\
(\mathrm{N}=43)\end{array}$ & $\begin{array}{l}\text { Control liver cohort (metastatic liver tumors, } \\
\qquad N=8)\end{array}$ \\
\hline Age & $66.5 \pm 7.8$ & $66.4 \pm 7.9$ & $66.5 \pm 7.9$ & $60.0 \pm 13.5$ \\
\hline \multicolumn{5}{|l|}{ Gender } \\
\hline Male & $35(74.4 \%)$ & $32(72.7 \%)$ & $32(74.4 \%)$ & $4(50.0 \%)$ \\
\hline Female & $12(25.5 \%)$ & $12(27.3 \%)$ & $11(25.6 \%)$ & $4(50.0 \%)$ \\
\hline \multicolumn{5}{|l|}{ Viral infection status } \\
\hline HBV infection & - & - & - & - \\
\hline HCV infection & + & + & + & - \\
\hline \multicolumn{5}{|l|}{ Liver resection procedure } \\
\hline Lobular/extended lobular & 5 & 5 & 5 & N/A \\
\hline Segmental/partial & 42 & 39 & 38 & \\
\hline Platelet count $\left(10^{4} / \mu \mathrm{l}\right)$ & $12.9 \pm 4.3$ & $13.0 \pm 4.2$ & $12.8 \pm 4.2$ & $19.3+3.6^{*}$ \\
\hline Alanine aminotransferase (IU/I) & $51.0 \pm 31.7$ & $51.0 \pm 32.0$ & $51.7 \pm 33.1$ & $19.7+7.7^{*}$ \\
\hline Total bilirubin (mg/dl) & $0.80 \pm 0.34$ & $0.76 \pm 0.30$ & $0.80 \pm 0.35$ & $0.85+0.37$ \\
\hline Prothrombin time (\%) & $78.3 \pm 13.1$ & $78.3 \pm 12.9$ & $78.1 \pm 13.3$ & $91.1+14.5$ \\
\hline Indocyanine green (\%) & $18.4 \pm 8.3$ & $18.5 \pm 8.3$ & $18.3 \pm 8.4$ & $11.0+5.3$ \\
\hline$\alpha$-Fetoprotein (ng/ml) & $319 \pm 924$ & $339 \pm 952$ & $345 \pm 962$ & N/A \\
\hline Tumor diameter (cm) & $2.90 \pm 1.30$ & $2.97 \pm 1.35$ & $2.95 \pm 1.37$ & N/A \\
\hline Number of tumors & $1.30 \pm 0.50$ & $1.32 \pm 0.52$ & $1.30 \pm 0.51$ & N/A \\
\hline \multicolumn{5}{|l|}{ Portal vein invasion } \\
\hline Present & $5(10.6 \%)$ & $5(11.4 \%)$ & $5(11.6 \%)$ & N/A \\
\hline Absent & $42(89.4 \%)$ & 39 (88.6\%) & $38(88.4 \%)$ & N/A \\
\hline \multicolumn{5}{|l|}{ Tumor stage } \\
\hline 1 & 9 (19.1\%) & $9(20.5 \%)$ & $8(18.6 \%)$ & N/A \\
\hline$\|$ & $25(53.2 \%)$ & $23(52.3 \%)$ & $24(55.8 \%)$ & N/A \\
\hline III & $12(25.5 \%)$ & $11(25.0 \%)$ & $10(23.3 \%)$ & N/A \\
\hline IV & $1(2.1 \%)$ & $1(2.3 \%)$ & $1(2.3 \%)$ & N/A \\
\hline \multicolumn{5}{|l|}{ Tumor differentiation } \\
\hline Well differentiated & $8(17.0 \%)$ & $8(18.2 \%)$ & $6(14.0 \%)$ & N/A \\
\hline Moderately differentiated & $31(66.0 \%)$ & $28(63.6 \%)$ & $29(67.4 \%)$ & N/A \\
\hline Poorly differentiated & $8(17.0 \%)$ & $8(18.2 \%)$ & $8(18.6 \%)$ & N/A \\
\hline \multicolumn{5}{|l|}{ Fibrosis score } \\
\hline Fo & $0(0 \%)$ & $0(0 \%)$ & $0(0 \%)$ & $8(100 \%)^{*}$ \\
\hline F1 & $3(6.4 \%)$ & $3(6.8 \%)$ & $3(7.0 \%)$ & $0(0 \%)$ \\
\hline F2 & $6(12.8 \%)$ & 5 (11.4\%) & $6(14.0 \%)$ & $0(0 \%)$ \\
\hline F3 & 14 (29.8\%) & $14(31.8 \%)$ & 12 (27.9\%) & $0(0 \%)$ \\
\hline F4 & $24(51.1 \%)$ & 22 (50.0\%) & $22(51.2 \%)$ & $0(0 \%)$ \\
\hline
\end{tabular}

Data shown are mean \pm standard deviation. N/A, data is not available. Asterisk $\left(^{*}\right)$ denotes statistical difference from other groups (ANOVA, $\left.\mathrm{p}<0.05\right)$.

primary tumor in a liver that is cirrhotic due to concomitant disease [20]. The molecular signatures of the tumor and its surrounding tissue, obtained through gene expression, proteomics or other means of profiling, are expected to reflect the causes of recurrence. Thus, an important challenge is to develop better understanding of the biological characteristics of the tumor and the surrounding tissue and to use this information to predict the outcomes.

Indeed, gene expression profiling has been instrumental in tackling these challenges and it has been shown that microarray analysis of the tumor samples can be used for prediction of clinical outcomes in subjects with HCC [21-23]. At the same time, others have reported no success with tumor-based transcription profiling [12]. Despite the fact that many clinical variables from tumors such as number of tumors, tumor stage, and tumor size were significantly associated with recurrence (Table 2), univariable feature selection with Cox scores did not identify robust markers of tumor recurrence in the context of leave-one-out cross-validation either in the entire cohort or in the sub-cohort of the early 
Table 2 Univariate analysis of clinico-pathological prognostic markers associated with recurrence-free survival in patients with HCC.

\begin{tabular}{|c|c|c|c|c|c|c|}
\hline & \multicolumn{3}{|c|}{ Nontumoral HCC Cohort $(\mathrm{N}=44)$} & \multicolumn{3}{|c|}{ Tumor HCC Cohort $(\mathrm{N}=43)$} \\
\hline & Risk ratio & $95 \% \mathrm{Cl}$ & P-value & Risk ratio & $95 \% \mathrm{Cl}$ & P-value \\
\hline Tumor stage & 2.48 & $1.39-4.51$ & 0.002 & 2.54 & $1.41-4.70$ & 0.002 \\
\hline Tumor diameter & 1.59 & $1.17-2.09$ & 0.005 & 1.65 & $1.19-2.24$ & 0.004 \\
\hline Number of tumors & 2.41 & $1.17-4.68$ & 0.02 & 2.10 & $0.99-4.19$ & 0.05 \\
\hline Platelet count & 0.95 & $0.87-1.03$ & 0.22 & 0.97 & $0.89-1.06$ & 0.53 \\
\hline Sex [male] & 1.44 & $0.67-3.43$ & 0.36 & 1.62 & $0.74-4.08$ & 0.24 \\
\hline Indocyanine green & 1.02 & $0.97-1.06$ & 0.40 & 1.01 & $0.97-1.05$ & 0.56 \\
\hline Prothrombin time & 0.99 & $0.97-1.02$ & 0.45 & 0.99 & $0.97-1.02$ & 0.40 \\
\hline Portal vein invasion [present] & 1.41 & $0.33-4.06$ & 0.59 & 1.42 & $0.34-4.08$ & 0.59 \\
\hline Liver resection procedure [lobular] & 1.14 & $0.62-1.82$ & 0.65 & 1.13 & $0.61-1.81$ & 0.66 \\
\hline Total Bilirubin & 1.26 & $0.42-3.24$ & 0.66 & 1.35 & $0.50-3.19$ & 0.54 \\
\hline Fibrosis score & 0.96 & $0.65-1.49$ & 0.86 & 0.92 & $0.63-1.38$ & 0.67 \\
\hline Tumor differentiation [moderate-poor] & 1.08 & $0.47-2.93$ & 0.86 & 0.84 & $0.35-2.49$ & 0.73 \\
\hline Age & 1.00 & $0.96-1.05$ & 0.90 & 1.02 & $0.97-1.07$ & 0.53 \\
\hline Alanine aminotransferase & 1.00 & $0.99-1.01$ & 0.96 & 1.00 & $0.99-1.01$ & 0.94 \\
\hline$\alpha$-Fetoprotein & 1.00 & $1.00-1.00$ & 0.99 & 1.00 & $1.00-1.00$ & 0.90 \\
\hline
\end{tabular}

Patients with HCV-associated HCC were divided into two cohorts based on the availability of mRNA samples. Univariate Cox proportional hazard model analysis was used to assess relative risk for each clinical variable. Relative risk $>1$ identifies shortened recurrence-free survival. Significant variables are highlighted in bold.

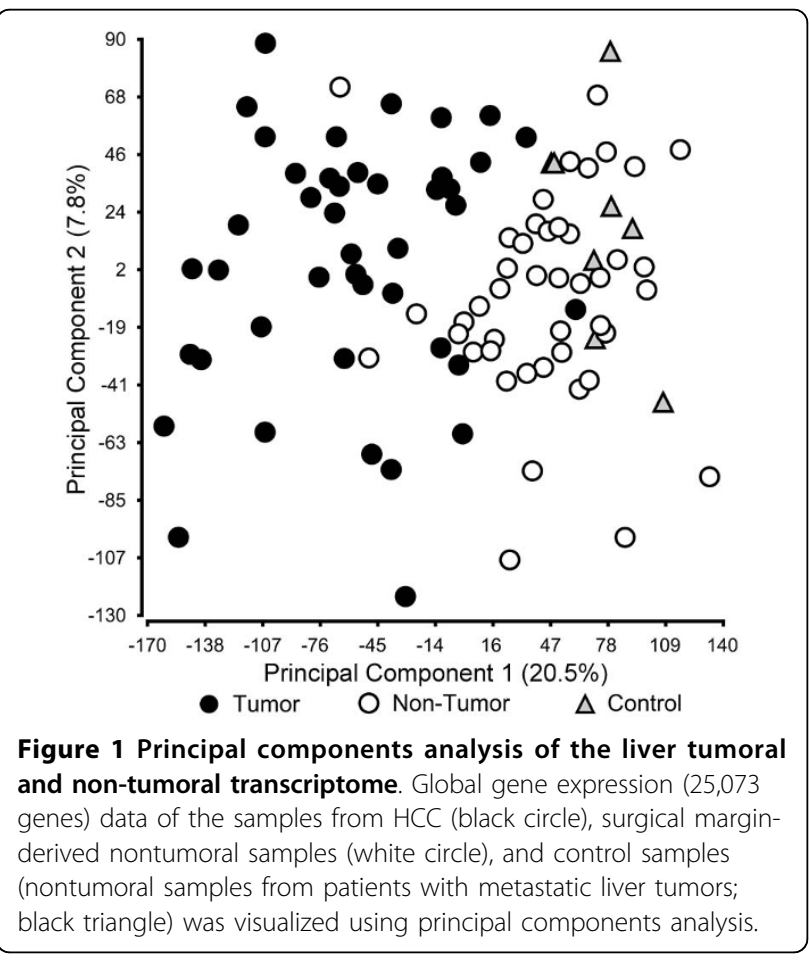

( $<1$ year) recurring subjects (data not shown). Possible reasons for our inability to create a predictor with tumor samples alone may include, among other factors, small sample size compounded by the considerable heterogeneity of gene expression between tumor samples (Additional file 2, Figure S2). However, our result is similar to the observations of Hoshida et al. [12] who also were unable to establish a tumor-derived predictor even with a much larger (hundreds of subjects) cohort. Although these results do not negate the value of tumor-derived expression profiling in predicting the outcomes of HCC, the data suggest that, at least in the cohort of $\mathrm{HCV}$-positive $\mathrm{HCC}$, tumor profiling alone may have limited value with regards to RFS.

Nonetheless, to further characterize the pathobiology of HCC recurrence we applied the gene set analysis algorithm to tumor-derived gene expression data in order to determine whether biologically-relevant gene sets that correlate with RFS exist. There were 1,497 gene sets based on 25,073 transcripts and a Cox score was generated for each gene set. Based on the nearest template prediction and leave-one-out cross-validation (i.e., independent prediction of each sample), several topranked (based on Cox score) gene sets were found to be predictive of tumor recurrence (Additional file 2, Figure S3). The gene set analysis has great value for assessment of the molecular profiles of the aggressively recurring tumors and may improve our understanding of the underlying biology that may be associated with the poor outcomes. Indeed, the most predictive gene set-based survival signature from HCC samples (Additional file 1, Table S4) contains a number of known key cancer-promoting pathways, such as mTOR [24], TGF $\beta$ [17], DNA damage response [25], hypoxia [26], histone deacetylase [27], and MET [28]. This data suggests that key tumorrelated genes may not only be involved in tumorigenesis, but may also be important for recurrence and thus can be used for selection of the subjects with poor prognosis. 
Nontumoral tissue-derived gene expression profiles and recurrence-free survival in $\mathrm{HCV}$-associated $\mathrm{HCC}$

Tumor-derived gene expression profiles did not produce a robust classifier of RFS in this cohort. This result led us to consider whether the surrounding liver tissue, not the tumor itself, may yield a molecular predictor associated with HCC recurrence. Indeed, several previous studies have shown that nontumoral tissue profiling in HCC holds great promise with regards to predicting clinical outcomes [11,12,29]. Forty-four nontumoral samples (94\% of the entire subject cohort) yielded highquality gene expression profiles. When both early- and late-recurrence samples were analyzed together, signatures composed of up to 18 top-ranked genes that correlate with disease-free survival were derived (Figure 2). The strongest signature (Figure 2B, $\mathrm{p}=0.02$ based on prediction during cross-validation) with the fewest number of genes is comprised of 14 genes (Figure 2C).

It has been suggested that distinct gene expression signatures may exist for prediction of early and late recurrence [15]. This is exemplified by the fact that little overlap exists between nontumoral tissue-derived signatures for predicting metastasis-related early recurrence [29], as opposed to those predictive of risk for the late recurrence [12]. Since late recurrence in $\mathrm{HCV}$-infected subjects is likely to be through de novo formation of tumors in the diseased liver, we selected a sub-cohort of 28 patents with late ( $>1$ year) recurrence and repeated the cross-validation procedure at both gene and gene set level. Even though a more traditional cut-off for "late" recurrence is 2 years, recent meta-analysis of HCC molecular subclasses suggests that 1 year time point may represent a more biologically-defined cut-off [19].

When individual transcript-level data was used, very strong predictive signatures could be derived for up to 91 genes (Additional file 2, Figure S4; Additional file 1, Table S5). All of the predictors composed of less than 38 genes were highly significant with 11 top-ranked genes producing the strongest signature (Figures $3 \mathrm{~A}-\mathrm{C}$, $\mathrm{p}<0.0001$ based on cross-validation, $0 \%$ error rate). Importantly, the genes identified as the strongest predictors of RFS that were over expressed in subjects with fastest recurrence have been previously implicated in tumor pathogenesis. For example, megakaryoblastic leukemia factor (MKL)1 has been shown to be required for TGF $\beta 1$ stimulation of alpha-smooth muscle actin expression [30], a process which promotes liver fibrosis and cirrhosis. TNKS1BP1 is a tankyrase 1-binding protein and it is known that tankyrase 1 upregulation leads to enhanced access of telomerase to telomeres and promotes clonal expansion of cancer cells [31]. Scaffold attachment factor B (SAFB) is playing a role in transcriptional repression and RNA splicing, and has been shown to be important in numerous cancer-related
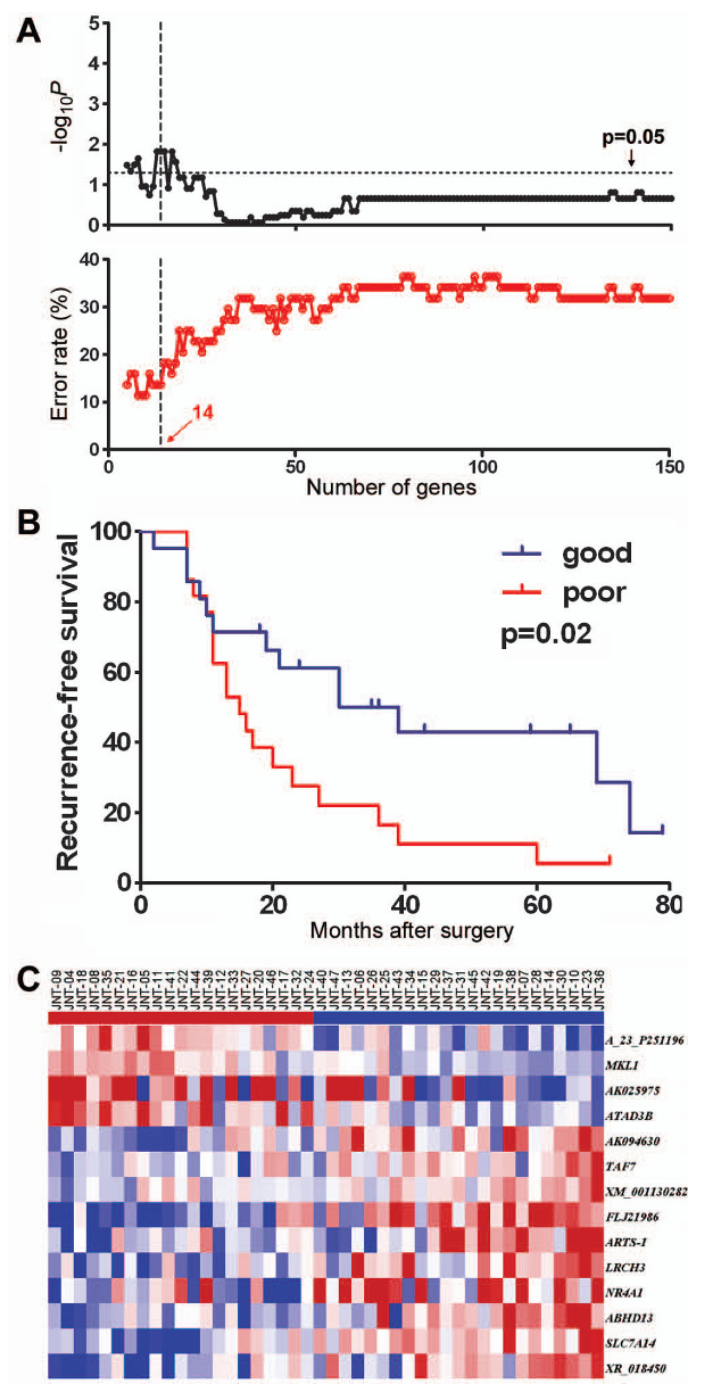

Figure 2 Recurrence-free survival analysis in the full cohort of HCC patients $(\mathrm{N}=44)$ using gene expression in nontumoral samples. Expression of 25,073 genes was used as a variable in Cox proportional hazard model. The power of recurrence-free survival prediction for the top-ranked (based on Cox score) genes was assessed using the Nearest Template Prediction (NTP) algorithm and cross-validated with a leave-one-out procedure. (A) Log-rank P-value and error rate of prediction for up to 150 top-ranked genes. (B) Recurrence-free survival curves based of the prediction made using 14 top-ranked genes. (C) Heat map of the 14 gene-based predictors. Red and blue indicate higher or lower than median gene expression, respectively.

cellular processes including cell growth, stress response, and apoptosis [32]. Protein phosphatase 2, regulatory subunit B, gamma isoform (PPP2R5C) was shown to function as a tumor suppressor gene. Upon DNA damage, a complex including PPP2R5C and p53 is formed which leads to dephosphorylation of p53, induction of the p53 transcriptional target p21, and the inhibition of cell proliferation [33]. 


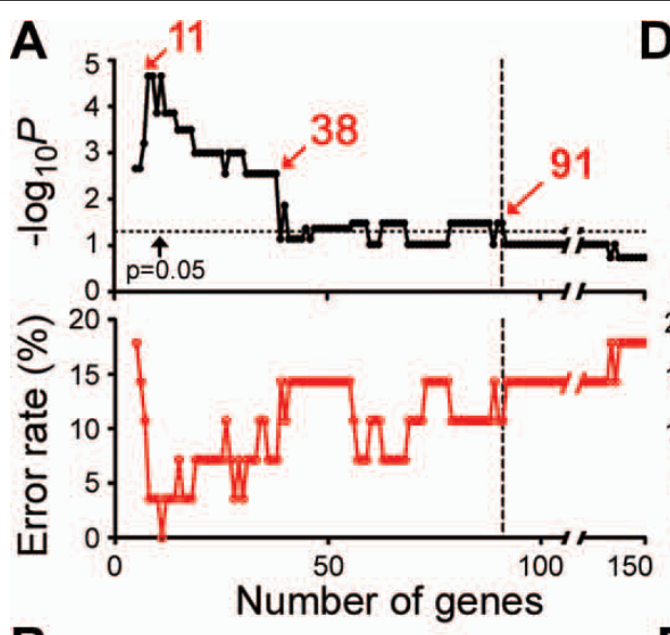

B

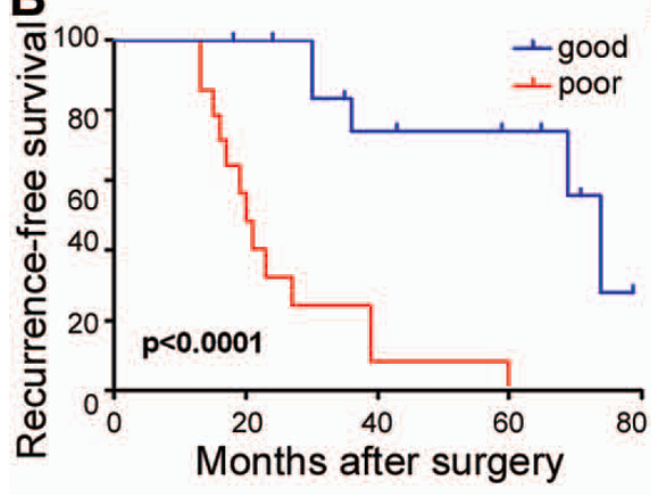

C

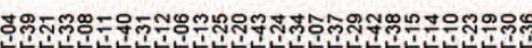

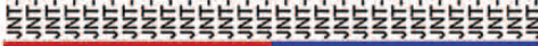

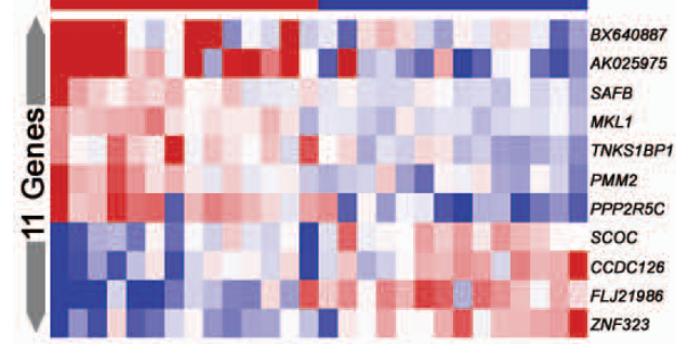

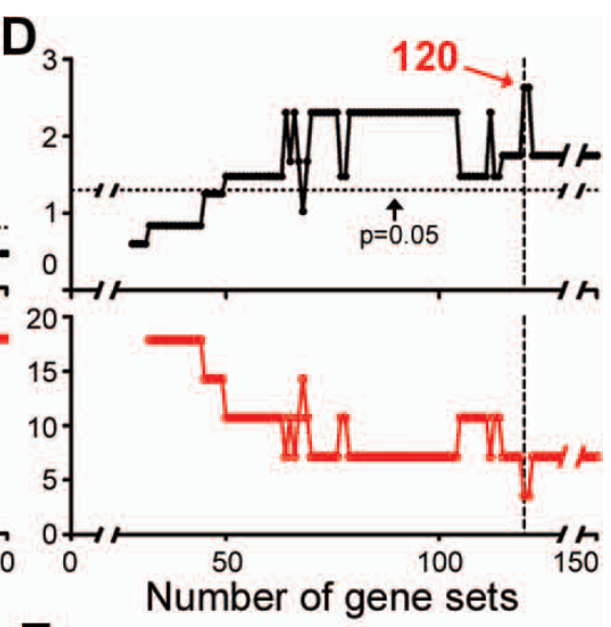

E

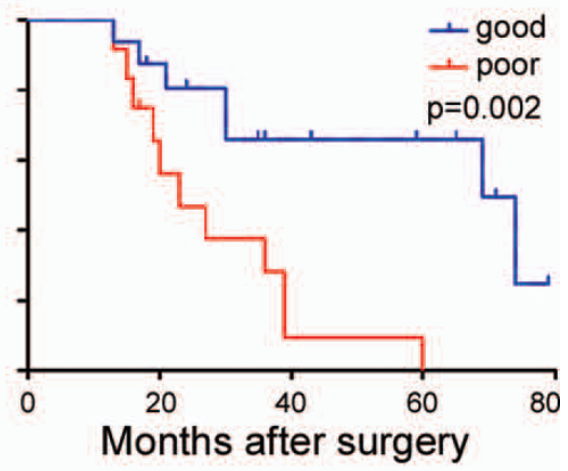

$\mathbf{F}$

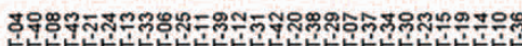

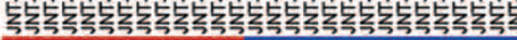

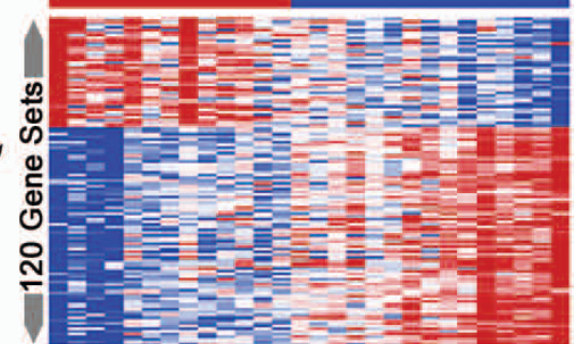

Figure 3 Recurrence-free survival analysis in HCC patients with late ( $>1$ year) recurrence using gene expression in nontumoral samples. (A and D) Logrank P-value and error rate of prediction for up to 150 top-ranked genes or gene sets. (B and E) Recurrence-free survival curves based of the prediction made using top-ranked genes (11) or gene sets (120), respectively (see Additional file 1, Tables S5-6 for the lists). ( $C$ and F) Heat map of the gene-, or gene set-based predictors. Red and blue indicate higher or lower than median gene expression, respectively.

Multivariable analysis of the nontumoral tissue-derived gene expression signatures of recurrence-free survival in HCV-associated HCC

The poor-prognosis late recurrence signature composed of 11, 38 or 91 top-ranked genes (Figure 3A), along with clinical variables available for the cohort of 28 subjects with recurrence $>1$ year, was analyzed by univariable Cox-proportional hazard model. Each of the gene expression-based poor-prognosis signatures, tumor diameter and the number of tumors were significantly associated with risk of recurrence in a univariable analysis (Table 3). Next, we examined the signatures in context of the clinical variables that were significant in our study and are also generally accepted as indicative of poor prognosis for recurrence of HCC. Importantly, a multivariable analysis (based on independent prediction of each sample using the cross-validation procedure as detailed above) of each set of top-ranked gene lists 
Table 3 Association of gene expression poor-prognosis signature and clinical variables with late ( $>1$ year) recurrence from univariate or multivariate analyses.

\begin{tabular}{|c|c|c|c|}
\hline Variable & $\begin{array}{l}\text { Risk } \\
\text { ratio }\end{array}$ & $95 \% \mathrm{Cl}$ & P-value \\
\hline \multicolumn{4}{|l|}{$\begin{array}{l}\text { Univariate Cox proportional hazard } \\
\text { model }\end{array}$} \\
\hline RFS signature (11 genes) & 3.13 & $1.75-6.58$ & $<0.0001$ \\
\hline RFS signature (38 genes) & 2.05 & $1.24-3.54$ & 0.005 \\
\hline RFS signature (91 genes) & 1.64 & $1.02-2.71$ & 0.04 \\
\hline Tumor diameter $[\mathrm{cm}]$ & 2.19 & $1.20-3.95$ & 0.01 \\
\hline Number of tumors & 4.28 & $\begin{array}{l}1.24- \\
13.39\end{array}$ & 0.02 \\
\hline Platelet count $\left[10^{4} / \mu \mathrm{L}\right]$ & 0.91 & $0.80-1.02$ & 0.11 \\
\hline Portal vein invasion [present] & 0.00 & $0.00-1.93$ & 0.15 \\
\hline Total bilirubin [mg/dL] & 1.54 & $0.37-4.97$ & 0.52 \\
\hline Age & 1.02 & $0.96-1.10$ & 0.53 \\
\hline Alanine aminotransferase [IU/L] & 0.99 & $0.97-1.01$ & 0.55 \\
\hline Indocyanine green [\%] & 1.02 & $0.95-1.08$ & 0.56 \\
\hline Prothrombin time [\%] & 0.99 & $0.96-1.03$ & 0.64 \\
\hline Liver resection procedure [lobular] & 1.16 & $0.46-2.21$ & 0.71 \\
\hline Fibrosis score & 0.93 & $0.52-1.72$ & 0.80 \\
\hline$\alpha$-Fetoprotein $[\mathrm{ng} / \mu \mathrm{L}]$ & 1.00 & $1.00-1.00$ & 0.85 \\
\hline Tumor differentiation [moderate-poor] & 0.91 & $0.32-3.27$ & 0.87 \\
\hline Sex [Male] & 0.93 & $0.35-2.71$ & 0.89 \\
\hline \multicolumn{4}{|l|}{$\begin{array}{l}\text { Multivariate Cox proportional hazard } \\
\text { model }\end{array}$} \\
\hline RFS signature ( 11 genes) & 3.38 & $1.75-7.77$ & 0.0001 \\
\hline Number of tumors & 8.55 & $\begin{array}{r}1.87- \\
46.69\end{array}$ & 0.006 \\
\hline Tumor diameter $[\mathrm{cm}]$ & 1.68 & $0.92-3.19$ & 0.09 \\
\hline RFS signature (38 genes) & 1.84 & $1.08-3.23$ & 0.02 \\
\hline Number of tumors & 4.59 & $\begin{array}{l}1.23- \\
16.11\end{array}$ & 0.02 \\
\hline Tumor diameter $[\mathrm{cm}]$ & 2.12 & $1.15-4.03$ & 0.02 \\
\hline RFS signature (91 genes) & 1.53 & $0.93-2.60$ & 0.09 \\
\hline Number of tumors & 3.58 & $0.98-12.3$ & 0.05 \\
\hline Tumor diameter $[\mathrm{cm}]$ & 2.46 & $1.30-4.70$ & 0.006 \\
\hline
\end{tabular}

Cox proportional hazard model analysis was used to assess relative risk for each clinical variable or RFS gene expression signature. Relative risk $>1$ identifies shortened recurrence-free survival. Significant variables are highlighted in bold font.

combined with two significant clinical variables (number of tumors and tumor diameter) showed that the late recurrence signatures composed of 11 and 38 genes, but not 91 genes, remained significant.

\section{Molecular predictors of tumor recurrence: clinical diagnostic tools or keys to tumor biology?}

Identification of gene expression signatures as predictors of the disease outcome has been a very active area of clinical cancer research [15,34]. The gene expression signatures outperform conventional clinicopathologic criteria in high-risk patients and help differentiate treatment options by identifying low-risk individuals [35]. Interestingly, while the signatures usually address the same clinical question, there is only little or no overlap in gene lists derived from different cohorts $[15,36]$. Most of the individual studies, including our work, are relatively small in size which may limit the potential for direct translation of the molecular signatures to the individual cancer patient management. To address this challenge, large comprehensive meta-analyses integrating both the gene expression and clinicopathologic data from multiple studies have been conducted for breast $[35,36]$, lung [34] and other cancers.

Indeed, a comparison of the applicability of the poorprognosis signatures from this study to the independent HCC cohort is the ultimate test for the 'biological' validation of the findings. There are two published microarray datasets that profiled nontumoral tissues from $\mathrm{HCC}$ subjects as potentially suitable for validation $[11,12]$ which may be used for cross-study validation. Unfortunately, the microarray used by Okamoto et al. [11] lacks comprehensive publicly accessible annotation (Agilent Technologies, personal communications) rendering any meaningful comparison between studies futile. The second study by Hoshida et al. [12] was conducted on formalin-fixed samples and used a specialized microarray containing $\sim 6,000$ probes. Not only there is only limited ( $20 \%)$ overlap in transcripts between the Illumina array (GPL5474, http://www.ncbi.nlm.nih.gov/geo/) and the whole human genome ( 44,000 probes) Agilent array used in our work, but also the clinico-pathological variables of the patient cohort used by the authors have not been released to the public (personal communication). Thus, while the cross-study comparisons shall remain as the goal for validation of the findings reported on independent cohorts and array platforms, greater degree of collaboration may be needed in the field of liver tumor biology.

While the molecular signatures generated in this study may not be clinic-ready without additional meta-analysis once the data becomes publicly available, we posit that late recurrence predictive genes may provide new insights into the oncogenic pathways for HCC development in cirrhotic patients. By examining the biological roles of the predictive genes it may be possible to better understand the process of field cancerization in HCC $[37,38]$ and a transition from cirrhosis to HCC. First, functional pathway analysis was performed using 91 top-ranked genes (Additional file 2, Figure S4; Additional file 1, Table S5) which comprise a comprehensive poor-prognosis signature in nontumoral tissues from subjects with late ( $>1$ year) recurrence (Figure 4 and Additional file 1, Table S7). Interestingly, hepatic nuclear factor (HNF) $4 \alpha$ - and interferon (IFN) $\gamma$-centered 
interactomes are top two most significant and interconnected networks (Figure 4A). Expression of HNF4 $\alpha$ is an important determinant of HCC progression and the development of aggressive tumor phenotypes in mice [39], while the T-helper 1 inflammatory cells characterized by IFN $\gamma$ secretion predominate in the liver during chronic HCV infection and are particularly important in disease progression [40]. The TP53-centered interactome is the top third significant network (Figure 4B) and the role of TP53 tumor suppressor pathway is widely recognized as significant contributors to liver carcinogenesis [41].
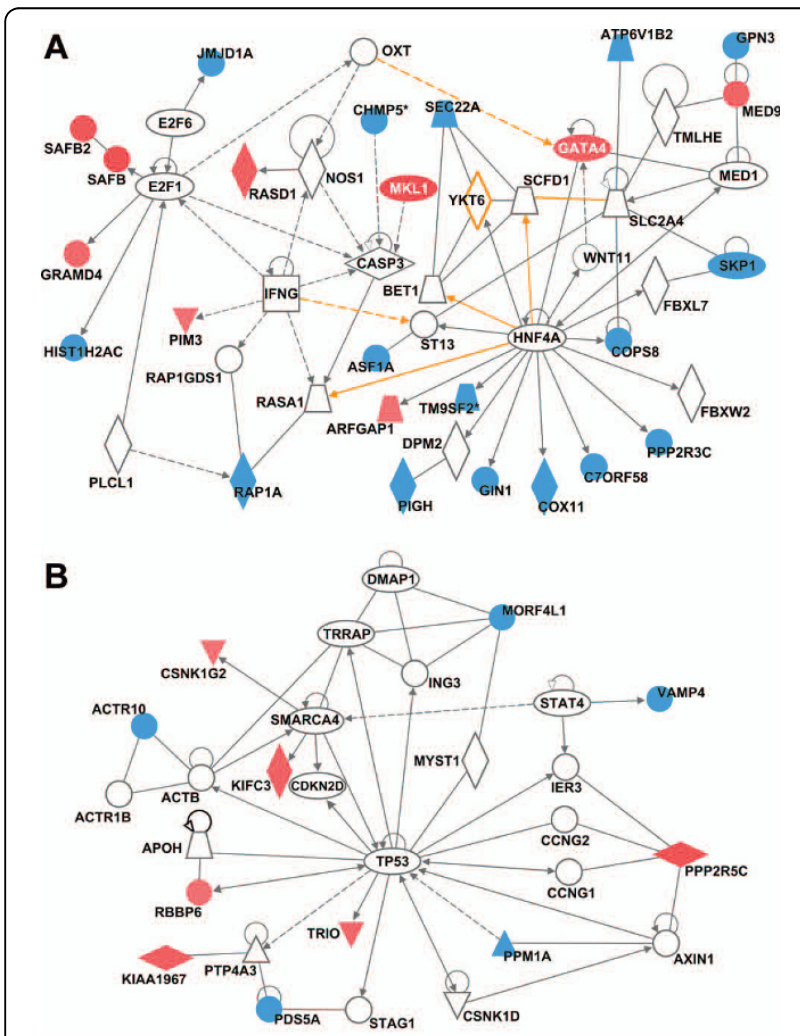

Figure 4 Molecular networks representing the RFS gene expression signature (91 genes) in HCC patients with late $(>1$ year) recurrence. See Additional file 1, Table $\mathrm{S} 7$ for a complete list of significant networks. (A) HNF4 $\alpha$ - and IFN $\gamma$-centered interactomes are top two most significant and interconnected (orange arrows) networks. (B) TP53-centered interactome is the top third significant network. Red and blue colors indicate positive or negative correlation, respectively, of gene expressions to recurrence-free survival based on Cox score. Ellipse, square, triangle, trapezoid, lozenge and circle represent transcription regulator, cytokine, kinase, transporter, enzyme and other molecules, respectively. Red and white represent molecules up-regulated in tumor samples compared to control and molecules incorporated into the network, respectively. Arrows connecting molecules indicate one molecule acts on another and lines indicate one binds to another. Dashed arrows or lines indicate indirect interactions of two molecules.
Second, gene set analysis was used to evaluate the data from the sub-cohort of late ( $>1$ year) recurrence subjects (Figures 3D-F). A number of top-ranked gene sets can serve as predictors (Additional file 1, Table S6) with the profile composed of 120 gene sets being most significant $(p=0.002)$ with the smallest error rate. Importantly, the gene set-based signature is overabundant in upregulated inflammation- (e.g., nuclear factor- $\kappa \mathrm{B}$, tumor necrosis factor), oxidative stress-, and carcinogenesis- (MYC, MAP kinase) related pathways, and down-regulated cell cycle control-related and protesome pathways. Interestingly, there appears to be great overlap in gene sets associated with poor prognosis outcome between nontumoral tissue and tumors (see Additional file 2, Figure S3, Additional file 1, Table S4) which further supports the theory of field cancerization and progression from cirrhosis to HCC.

\section{Conclusions}

Our study demonstrates that microarray analysis of the nontumoral tissues from subjects with $\mathrm{HCV}$-associated HCC delivers novel molecular signatures of RFS, especially among the late-recurrence subjects. Since late recurrence is likely a de novo tumor occurrence in HCV-associated cirrhosis, the biopsy-based gene expression analysis in cirrhotic patients may be a useful method to stratify the risk of tumor occurrence and determine a need for aggressive patient screening. While challenges remain in validating the signatures and translating the findings to clinical practice, we posit that gene expression-based signatures provide important insights into pathobiology of HCC recurrence.

\section{Methods}

\section{Subjects, specimen collection and study design}

Forty-seven subjects with HCV-associated HCC who underwent complete removal of the tumor at the University of Yamanashi Hospital (Yamanashi Prefecture, Japan) between 2000 and 2007 were enrolled in this study. All of the subjects tested positive for HCV and negative for hepatitis $B$ virus. The presence and identification of the hepatitis virus was determined by one or more of the following techniques: (i) presence of anti$\mathrm{HCV}$ and anti-HBV reactive serum proteins, (ii) reverse transcription-PCR for serum HCV-RNA, or (iii) branched DNA-HCV probe assay. Following the tumor resection procedure and recovery after surgery, each subject visited the ambulatory care clinic for additional tests monthly. Serum $\alpha$-fetoprotein levels were measured every month. In addition, ultrasonography or computed tomography of the liver was performed every 3 and 6 months, respectively. Eight HCV- and HBV-free subjects who were diagnosed with metastatic liver tumors that required surgical resection were enrolled as 
the control group and nontumoral tissue samples were obtained. All samples were frozen in liquid nitrogen immediately following surgery and kept as $-80^{\circ} \mathrm{C}$ until processed. Informed consent was received from each subject in the study and the study protocol conforms to the ethical guidelines of the 1975 Declaration of Helsinki as reflected in an a priori approval by the Institutional Board on Ethics for Human Science at the University of Yamanashi. All available clinical data for the subjects enrolled in this study are summarized in Table 1. RFS data are provided in Additional file 1, Table S1.

\section{Statistical analysis}

JMP software (ver. 6.0, SAS institute, Cary, NC) was used for statistical analysis of the clinical and molecular (see below) variables. For univariable analysis, two-sample t-test, linear regression, or Logrank test statistical procedures were used to assess what endpoints could be used for predicting prognosis of HCC subjects after surgery. For multivariable analysis, the Cox proportional hazard model was used to calculate hazard ratio and pvalue of each parameter. A $\mathrm{p}$ value of less than 0.05 was considered significant.

\section{Microarray analysis}

Total RNA was isolated from tumor and nontumoral liver samples ( $30 \mathrm{mg})$ with an RNeasy plus mini kit (Qiagen, Valencia, CA) as detailed by the manufacturer. High quality RNAs was obtained from 43 tumor and 44 nontumoral samples (40 tumor and nontumoral RNA samples were from the same subjects). For microarray analysis, a balanced design was used. Each batch of arrays (Agilent Technologies, Wilmington, DE; whole human genome microarray, cat.\#G4112F) was balanced with regards to the tumor stage and RFS and contained both inter-batch and intra-batch replicates.

RNA samples were hybridized to arrays individually; none were pooled. RNA amplifications and labeling were performed using Low RNA Input Linear Amplification kits (Agilent Technologies). For hybridization, 1.2 $\mu \mathrm{g}$ of total RNA from each sample, or human reference RNA (Stratagene, La Jolla, CA; Cat\#740000) was amplified and labeled with fluorescent dye (Cy5 and Cy3, respectively). RNA labeling, microarray hybridization, washing and scanning were performed according to the manufacturer's instructions. Feature extraction, normalization of the raw data and data filtering were performed using the Agilent Feature Extraction software (v8.5, Agilent Technologies). Raw microarray data was archived in Gene Expression Omnibus (GSE17856) and is available to the public. The $\log _{2}$ ratio of $\mathrm{Cy} 5 / \mathrm{Cy} 3$ intensity was normalized using LOWESS smoothing to eliminate intensity bias of features. Transcripts with fewer than
$70 \%$ available data across samples were excluded from the analysis, reducing the probe list to 25,073 transcripts. Available data are defined as those probes that are neither saturated nor below the limit of quantification. Inter-batch normalization was carried out using Distance Weighted Discrimination procedure [42].

\section{Biological pathway analysis on tumor samples}

Differentially expressed genes between control and tumor sample groups were identified by using Significant Analysis of Microarray analysis [43]. Individual genes with false discovery rate $(\mathrm{FDR})=0$ in the univariable test under 1,000 random permutations were considered significant. Selected genes were analyzed using Ingenuity Pathways Analysis (v. 7.1; Ingenuity Systems, Redwood City, CA) to determine canonical pathways that are enriched among the significant transcripts. SAFEGUI [44] was used to compare gene expression level between HCC tumor samples and control nontumoral liver samples from subjects with metastatic liver tumors based on Gene Ontology (GO) biological process categories, or Kyoto Encyclopedia of Genes and Genomes (KEGG) pathways. P-values less than 0.01 were considered to be significant.

\section{Gene expression-based recurrence-free survival (RFS) predictor}

Tumor and adjacent nontumoral liver tissues were considered separately to define an RFS-predictive signature on each type of tissue. In each dataset, genes whose expression was associated with time to recurrence were selected by means of the high absolute Cox score and prediction analysis was performed by evaluating the expression status of the signature using the nearest template prediction method as described elsewhere[12] and implemented in GenePattern software (ver.3.1.1, http:// www.broad.mit.edu/cancer/software/genepattern/). The signatures were validated using a leave-one-out crossvalidation prediction procedure. In brief, each sample was left out one-by-one and a disease-free survival-correlated signature was deduced using remaining samples (selecting marker genes based on top-ranked highest absolute Cox score). A predicted class label was assigned to the left-out sample based on the closest "template" using the nearest template prediction algorithm. A maximum of 200 top-ranked genes were used to make predictions in each analysis. The outcome of cross-validation based prediction was assessed by Logrank test and the "error rate," defined here as the ratio of the number of subjects predicted incorrectly to that in the model. In parallel, the "gene set" analysis (GSA) was also used to determine the significance of pre-defined sets of genes with respect to an outcome variable, such as a RFS time. GSA package for R http://www-stat. 
stanford.edu/ tibs/GSA/ was utilized. The output from GSA was used to generate RFS predictors in the same manner as detailed above for gene-level data and validated using a leave-one-out cross-validation procedure.

\section{List of Abbreviations}

HCC: Hepatocellular Carcinoma; HCV: Hepatitis C Virus; FDR: False Discovery Rate; GO: Gene Ontology; KEGG: Kyoto Encyclopedia of Genes and Genomes; RFS: Recurrence-Free Survival; SAFE: Significance Analysis of Function and Expression.

Additional file 1: table S1. Clinical outcomes for each individual patient included in this study. table S2. Molecular networks (Ingenuity ${ }^{\oplus}$ pathways analysis) differentially modulated between control (nontumoral samples from patients with metastatic liver tumors) tissue and HCC tumors. table S3. GO categories and KEGG pathways that differ significantly between control and HCC tumor samples were identified using SAFE analysis (see Methods). table S4. Sixty seven top-ranked gene sets used for recurrence prediction analysis in tumor samples (see Figure $3 \mathrm{C}$ for the heat map). table S5. Ninety one top-ranked genes used for recurrence prediction analysis in nontumoral samples from late ( $>1$ year) recurrence subjects (see Figure $3 \mathrm{C}$ for the heat map). table S6. One hundred twenty topranked gene sets used for recurrence prediction analysis in nontumoral samples from late ( $>1$ year) recurrence subjects (see Figure $3 \mathrm{~F}$ for the heat map). table 57 . Molecular networks representing the recurrence-free survival gene expression signature (91 genes) in HCC patients with late (>1 year) recurrence.

Additional file 2: Figure S1. TGF $\beta$ 1- and MYC-centered interactomes are most significantly up-regulated molecular networks in HCC tumors (see Additional file 1, Table S2-3 for a full list of the significant pathways). Ellipse, square, triangle, trapezoid, lozenge and circle represent transcription regulator, cytokine, kinase, transporter, enzyme and other molecules, respectively. Red and white represent molecules up-regulated in tumor samples compared to control and molecules incorporated into the network, respectively. Arrows connecting molecules indicate one molecule acts on another and lines indicate one binds to another. Dashed arrows or lines indicate indirect interactions of two molecules. Figure S2. Analysis of the dissimilarity (Spearman's correlation) between gene expression profiles of the nontumoral (top) or tumoral (bottom) samples used in this study. Expression of 25,073 genes was used as a variable to create the heat map of each dissimilarity matrix. The color bar represents the degree of dissimilarity with red exhibiting high degree of dissimilarity and blue - high similarity. Figure S3. Recurrence-free survival analysis in HCC patients using gene set analysis in tumor samples. (A) Logrank P-value and error rate of prediction for up to 150 top-ranked gene sets. (B) Recurrence-free survival curves (Kaplan-Meier method) based of the prediction made using 67 top-ranked gene sets (Additional file 1, Table S4). Red and blue represent the subjects predicted to have poor or good recurrence-free survival, respectively. (C) Heatmap of the 67 gene set-based survival signature from tumor samples. Red and blue indicate high or low gene set score, respectively. Subjects ( $x$-axis) are sorted by means of cosine distance to 'poor' template, and gene sets (y-axis) are sorted by Cox score (Additional file 1, Table S4). Figure S4. Recurrence-free survival analysis in HCC patients with late (>1 year) recurrence using gene expression (91 genes) in nontumoral samples. (A) 91 genes selected based on log-rank P-value and error rate of prediction were used to construct recurrence-free survival curves (B). (C) Heat map of the gene-based survival signature with 91 genes.

\section{Acknowledgements}

The authors wish to acknowledge assistance from Atsushi Kawaguchi, PhD, Kurume University, Japan, for statistical consultations. Financial support for these studies was provided, in part, by grants from the National Institutes of Health: R01 AA016285 and R01 ES015241.

\section{Author details}

'Department of Environmental Sciences and Engineering, Gillings School of Global Public Health, University of North Carolina, Chapel Hill, NC, USA. ${ }^{2}$ Curriculum in Genetics, School of Medicine, University of North Carolina, Chapel Hill, NC, USA. ${ }^{3}$ First Department of Surgery, University of Yamanashi, Chuo, Yamanashi Prefecture, Japan.

\section{Authors' contributions}

MT participated in the design of the study, carried out the molecular and statistical analyses and drafted the manuscript; JP carried out the statistical analyses and participated in writing of the manuscript; HK, MM and HF designed the study, performed sample analyses and assisted with the development of the manuscript; IR designed the study, participated in the analyses of the data and was responsible for writing of the manuscript. All authors read and approved the final manuscript.

\section{Competing interests}

The authors declare that they have no competing interests.

Received: 13 January 2010 Accepted: 9 April 2010

Published: 9 April 2010

\section{References}

1. Caldwell S, Park SH: The epidemiology of hepatocellular cancer: from the perspectives of public health problem to tumor biology. J Gastroenterol 2009, 44(Suppl 19):96-101.

2. Llovet JM, Burroughs A, Bruix J: Hepatocellular carcinoma. Lancet 2003, 362:1907-1917.

3. SEER Cancer Statistics Review, 1975-2006. [http://seer.cancer.gov/csr/ 1975_2006/].

4. Kiyosawa K, Umemura T, Ichijo T, Matsumoto A, Yoshizawa K, Gad A, Tanaka E: Hepatocellular carcinoma: recent trends in Japan. Gastroenterology 2004, 127:S17-S26.

5. Jepsen P, Vilstrup H, Tarone RE, Friis S, Sorensen HT: Incidence rates of hepatocellular carcinoma in the U.S. and Denmark: recent trends. Int J Cancer 2007, 121:1624-1626.

6. Bruix J, Llovet JM: Major achievements in hepatocellular carcinoma. Lancet 2009, 373:614-616.

7. Llovet JM, Di Bisceglie AM, Bruix J, Kramer BS, Lencioni R, Zhu AX Sherman M, Schwartz M, Lotze M, Talwalkar J, et al: Design and endpoints of clinical trials in hepatocellular carcinoma. J Natl Cancer Inst 2008, 100:698-711.

8. Llovet JM, Ricci S, Mazzaferro V, Hilgard P, Gane E, Blanc JF, de Oliveira AC Santoro A, Raoul JL, Forner A, et al: Sorafenib in advanced hepatocellular carcinoma. N Engl J Med 2008, 359:378-390.

9. Minagawa M, Makuuchi M, Takayama T, Kokudo N: Selection criteria for repeat hepatectomy in patients with recurrent hepatocellular carcinoma. Ann Surg 2003, 238:703-710.

10. Adachi E, Maeda T, Matsumata T, Shirabe K, Kinukawa N, Sugimachi K, Tsuneyoshi M: Risk factors for intrahepatic recurrence in human small hepatocellular carcinoma. Gastroenterology 1995, 108:768-775.

11. Okamoto M, Utsunomiya T, Wakiyama S, Hashimoto M, Fukuzawa K, Ezaki T, Hanai T, Inoue H, Mori M: Specific gene-expression profiles of noncancerous liver tissue predict the risk for multicentric occurrence of hepatocellular carcinoma in hepatitis C virus-positive patients. Ann Surg Oncol 2006, 13:947-954

12. Hoshida Y, Villanueva A, Kobayashi M, Peix J, Chiang DY, Camargo A, Gupta S, Moore J, Wrobel MJ, Lerner J, et al: Gene expression in fixed tissues and outcome in hepatocellular carcinoma. N Engl J Med 2008, 359:1995-2004

13. Maki A, Kono H, Gupta M, Asakawa M, Suzuki T, Matsuda M, Fuiji H, Rusyn I: Predictive power of biomarkers of oxidative stress and inflammation in patients with hepatitis C virus-associated hepatocellular carcinoma. Ann Surg Oncol 2007, 14:1182-1190.

14. Tsuchiya M, Kono H, Matsuda M, Fujii $H$, Rusyn I: Protective effect of Juzen-taiho-to on hepatocarcinogenesis is mediated through the inhibition of Kupffer cell-induced oxidative stress. Int I Cancer 2008, 123:2503-2511. 
15. Wang XW, Thorgeirsson SS: Transcriptome analysis of liver cancer: ready for the clinic? J Hepatol 2009, 50:1062-1064.

16. Pang RW, Joh JW, Johnson PJ, Monden M, Pawlik TM, Poon RT: Biology of hepatocellular carcinoma. Ann Surg Oncol 2008, 15:962-971.

17. Matsuzaki K, Murata M, Yoshida K, Sekimoto G, Uemura Y, Sakaida N, Kaibori M, Kamiyama Y, Nishizawa M, Fujisawa J, et al: Chronic inflammation associated with hepatitis $C$ virus infection perturbs hepatic transforming growth factor beta signaling, promoting cirrhosis and hepatocellular carcinoma. Hepatology 2007, 46:48-57.

18. Kaposi-Novak P, Libbrecht L, Woo HG, Lee YH, Sears NC, Coulouarn C, Conner EA, Factor VM, Roskams T, Thorgeirsson SS: Central role of c-Myc during malignant conversion in human hepatocarcinogenesis. Cancer Res 2009, 69:2775-2782.

19. Hoshida Y, Nijman SM, Kobayashi M, Chan JA, Brunet JP, Chiang DY, Villanueva A, Newell P, Ikeda K, Hashimoto M, et al: Integrative transcriptome analysis reveals common molecular subclasses of human hepatocellular carcinoma. Cancer Res 2009, 69:7385-7392.

20. Chen YJ, Yeh SH, Chen JT, Wu CC, Hsu MT, Tsai SF, Chen PJ, Lin CH: Chromosomal changes and clonality relationship between primary and recurrent hepatocellular carcinoma. Gastroenterology 2000, 119:431-440.

21. Lee JS, Chu IS, Heo J, Calvisi DF, Sun Z, Roskams T, Durnez A, Demetris AJ, Thorgeirsson SS: Classification and prediction of survival in hepatocellular carcinoma by gene expression profiling. Hepatology 2004, 40:667-676.

22. Ho MC, Lin JJ, Chen CN, Chen CC, Lee H, Yang CY, Ni YH, Chang KJ, Hsu HC, Hsieh FJ, et al: A gene expression profile for vascular invasion can predict the recurrence after resection of hepatocellular carcinoma: a microarray approach. Ann Surg Oncol 2006, 13:1474-1484.

23. Yoshioka S, Takemasa I, Nagano H, Kittaka N, Noda T, Wada H, Kobayashi S, Marubashi S, Takeda Y, Umeshita K, et al: Molecular prediction of early recurrence after resection of hepatocellular carcinoma. Eur J Cancer 2009, 45:881-889.

24. Sabatini DM: mTOR and cancer: insights into a complex relationship. Nat Rev Cancer 2006, 6:729-734.

25. Hussain SP, Hofseth LJ, Harris CC: Radical causes of cancer. Nat Rev Cancer 2003, 3:276-285.

26. Hamaguchi T, lizuka N, Tsunedomi $R$, Hamamoto $Y$, Miyamoto $T$, lida M, Tokuhisa Y, Sakamoto K, Takashima M, Tamesa T, et al: Glycolysis module activated by hypoxia-inducible factor 1alpha is related to the aggressive phenotype of hepatocellular carcinoma. Int J Oncol 2008, 33:725-731.

27. Miura K, Taura K, Kodama Y, Schnabl B, Brenner DA: Hepatitis C virusinduced oxidative stress suppresses hepcidin expression through increased histone deacetylase activity. Hepatology 2008, 48:1420-1429.

28. Kaposi-Novak P, Lee JS, Gomez-Quiroz L, Coulouarn C, Factor VM, Thorgeirsson SS: Met-regulated expression signature defines a subset of human hepatocellular carcinomas with poor prognosis and aggressive phenotype. J Clin Invest 2006, 116:1582-1595.

29. Budhu A, Forgues M, Ye QH, Jia HL, He P, Zanetti KA, Kammula US, Chen Y, Qin LX, Tang ZY, et al: Prediction of venous metastases, recurrence, and prognosis in hepatocellular carcinoma based on a unique immune response signature of the liver microenvironment. Cancer Cell 2006, 10:99-111.

30. Elberg G, Chen L, Elberg D, Chan MD, Logan CJ, Turman MA: MKL1 mediates TGF-beta1-induced alpha-smooth muscle actin expression in human renal epithelial cells. Am J Physiol Renal Physiol 2008, 294 F1116-F1128.

31. Seimiya H, Muramatsu Y, Ohishi T, Tsuruo T: Tankyrase 1 as a target for telomere-directed molecular cancer therapeutics. Cancer Cell 2005, 7:25-37.

32. Garee JP, Oesterreich S: SAFB1's multiple functions in biological controllots still to be done! I Cell Biochem 2010, 109:312-319.

33. Shouse GP, Cai X, Liu X: Serine 15 phosphorylation of p53 directs its interaction with B56 gamma and the tumor suppressor activity of B56 gamma-specific protein phosphatase 2A. Mol Cell Biol 2008, 28:448-456.

34. Sotiriou C, Piccart MJ: Taking gene-expression profiling to the clinic: when will molecular signatures become relevant to patient care? Nat Rev Cancer 2007, 7:545-553.

35. Parker JS, Mullins M, Cheang MC, Leung S, Voduc D, Vickery T, Davies S, Fauron $\mathrm{C}, \mathrm{He} X$, Hu Z, et al: Supervised risk predictor of breast cancer based on intrinsic subtypes. J Clin Oncol 2009, 27:1160-1167.

36. Desmedt C, Haibe-Kains B, Wirapati P, Buyse M, Larsimont D, Bontempi G, Delorenzi M, Piccart M, Sotiriou C: Biological processes associated with breast cancer clinical outcome depend on the molecular subtypes. Clin Cancer Res 2008, 14:5158-5165.

37. Lou C, Du Z, Yang B, Gao Y, Wang Y, Fang S: Aberrant DNA methylation profile of hepatocellular carcinoma and surgically resected margin. Cancer Sci 2009, 100:996-1004.

38. Utsunomiya T, Shimada M, Imura S, Morine Y, Ikemoto T, Mori M: Molecular signatures of noncancerous liver tissue can predict the risk for late recurrence of hepatocellular carcinoma. J Gastroenterol 2010, 45:146-152.

39. Lazarevich NL, Cheremnova OA, Varga EV, Ovchinnikov DA, Kudrjavtseva El, Morozova OV, Fleishman DI, Engelhardt NV, Duncan SA: Progression of HCC in mice is associated with a downregulation in the expression of hepatocyte nuclear factors. Hepatology 2004, 39:1038-1047.

40. Zeremski M, Petrovic LM, Talal AH: The role of chemokines as inflammatory mediators in chronic hepatitis $C$ virus infection. $J$ Viral Hepat 2007, 14:675-687.

41. Staib F, Hussain SP, Hofseth $\sqcup$, Wang XW, Harris CC: TP53 and liver carcinogenesis. Hum Mutat 2003, 21:201-216.

42. Benito M, Parker J, Du Q, Wu J, Xiang D, Perou CM, Marron JS: Adjustment of systematic microarray data biases. Bioinformatics 2004, 20:105-114.

43. Tusher VG, Tibshirani R, Chu G: Significance analysis of microarrays applied to the ionizing radiation response. Proc Natl Acad Sci USA 2001, 98:5116-5121.

44. Gatti DM, Sypa M, Rusyn I, Wright FA, Barry WT: SAFEGUI: resamplingbased tests of categorical significance in gene expression data made easy. Bioinformatics 2009, 25:541-542.

doi:10.1186/1476-4598-9-74

Cite this article as: Tsuchiya et al:: Gene expression in nontumoral liver tissue and recurrence-free survival in hepatitis $C$ virus-positive hepatocellular carcinoma. Molecular Cancer 2010 9:74.

\section{Submit your next manuscript to BioMed Central and take full advantage of:}

- Convenient online submission

- Thorough peer review

- No space constraints or color figure charges

- Immediate publication on acceptance

- Inclusion in PubMed, CAS, Scopus and Google Scholar

- Research which is freely available for redistribution
Ciomed Central 